\title{
Research on the Evolution of Health Information Behavior From a Chinese Perspective
}

\author{
Nian Ding \\ Wuhan University \\ dingnian@whu.edu.cn
}

\author{
Xiao Huang* \\ Wuhan University \\ 851195240@qq.com
}

\begin{abstract}
China has been undergoing a tremendous development in the reform of health system and it has great effects all the citizens and the nation as a whole. This paper aims to focus on the individuals from the aspect of information behavior. It is expected that the review on health information behavior could be conducted in a systematic way. Moreover, some statistical methods and software have been occupied in order to find out the entire progress of health information behavior. Specifically, both vertical and horizontal comparison have been conducted in this study, and scientometric methods have also be used. After a systematic and profound literature review, the whole progress has been explored and the main topics of great importance have been discovered. Moreover, highly cited papers and their relationship have also been revealed.
\end{abstract}

\section{Introduction}

As a leading-edge area, health information behavior has been the essential part for Information System and other related disciplines, such as Library and Information Science. In order to get a whole landscape of the evolution for this area, a literature study with a scientometric method was conducted. Health information behavior is an emerging interdisciplinary subject which combined both information science and technology. It involves theory and methodology in different fields, including Information System, Computer Science, Library and Information Science and Medicine. It is a promising direction for researchers since health issues have the greatest significance for people. It is definitely to obtain great concern and focus all the time. With the advent of new method and technology, such as AI and machine learning, tremendous changes happened in this area. As a result, this research intends to grasp the evolutionary progress of this area, and capture the latest tendency and valuable feature for related topics.
Moreover, it is intended to shed a light for the future research in this area.

With the ambitious health reform launched in 2009, China has been undertaking an unprecedented transformation in health system. Adherently, the number of published papers had a rapid increasing since then[1]. With the target of universal health coverage(UHC), overwhelming developments have been witnessed. China Series and themed issues of China have been published on Lancet, which indicated the significant role of Chinese Health Reform and Transformation.

This paper is oriented to the area of health information behavior. We want to have a clear picture about the whole developing progress of health information behavior in China. Accordingly, this study is going to analyze the evolutionary progress of the topic health information behavior from a Chinese perspective. To be specific, this study is supposed to analyze the papers which have Chinese scholars involved in. It is dedicated to reflect the research progress of health information behavior in a reasonable and profound way.

\section{Methods}

Keeping the mind the aim of this paper, we used Cortext (https://www.cortext.net/) and VOSview (http://www.vosviewer.com/) as the tool. Cortext is able to detect the evolution of topics based on keywords and present the results with good visualizations. The tool is suitable for the analysis of hot topics in a research field. VOSview is a software tool for constructing and visualizing bibliometric networks. Its core principle is "co-occurrence clustering". The tool is suitable for keyword cooccurrence cluster analysis and co-citation clustering analysis in a research field.

(1) Identify the domain of "health information behavior", especially researches concluding researchers from China. According to the research of well-known scholars in this field in China and other literature in the field, we found that there are many 
keywords related to the field of "health information behavior", including "online health information" "mhealth information" "health informative" "online health community" "online health communities" and so on.

(2) Data collection. The retrieval strategy is "TS=("online *health informati*" OR "online *health communit*" OR "*health informati*" OR "*health communit*") AND CU=China", which has been conducted on ISI Web of Science Core Citation Database. According to the search formula, we obtained a total of 1,252 papers. But these 1,252 papers are not all related to "health information behavior". Through further manual screening, we have a total of 412 papers in the field of "health information behavior".

(3) Data preprocessing. Obtain the information, keywords, abstracts, journals and other information of 412 documents that were clear in the previous process. Combine this information into a single file and adjust the file to make it readable by the analysis software.

(4) Data analysis. Cortext was used to analyze the subject evolution trends of these documents, using VOSview for keyword co-occurrence cluster analysis and co-citation analysis.

\section{Literature review}

\subsection{Analysis on the Number of Published Papers}

The number of papers published in health information behavior in China from 2004 to 2019 is shown in Figure 1. The first paper about this topic was published in 2004. The literature entitled "Quality and safety issues of web-based information about herbal medicines in the treatment of cancer" was published in the journal named COMPLEMENTARY THERAPIES IN MEDICINE, this research assesses the quality and safety of the information presented on the internet about medicinal herbs specifically in the field of cancer[2].

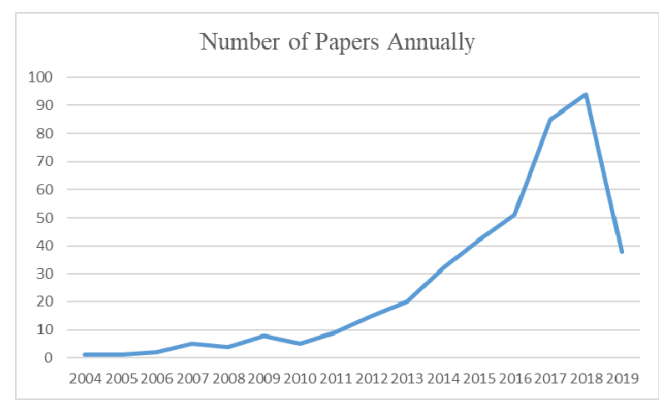

Figure.1. Number of papers from 2004 to 2019

It could be easily found that there is a smooth growth of the number of published papers each year. To be precise, there were no more than five papers published annually during the first five years. In 2009 , there was a small growth of the publications, which is consistent with the launching of the health reform in China. Since then, more and more researches have been achieved, and the growth seems to be extremely steady. Until now, there are 412 papers closely related to the topic health information behavior in the core databases of WOS. And they are the research object of this study.

\subsection{Evolution of Hot Topics}

Figure 2 is a map of the research evolution of this area based on keywords. Both persistency and transiency exist in the evolution of this area. This graph indicates some of the topics have been the focus for a long period, while some of them flashed just for a while.

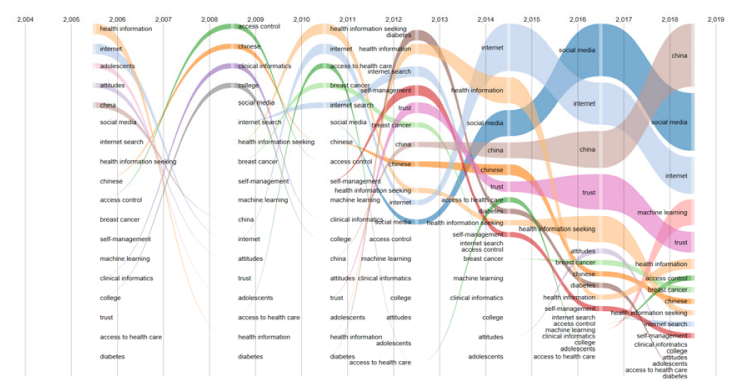

Figure.2. Research evolution of hot topics

There was a good start in 2004, when the first relevant paper was got in WOS. The Chinese scholar took part in the cooperative study in the field of health information behavior in 2014 for the first time. It is about the quality of web-based information about herbal medicines in the treatment of cancer. The result showed that most websites had low quality of information about medical herbs, and biomedical websites had better performance and higher score that commercial ones. At that time, no Chinese researchers had independent study in this field.

The year of 2005 could be deemed as the real beginning of Chinese research in health information behavior. In that year, an international conference was held in Chongqing, which was International Conference on Service Systems and Service Management. A group of Chinese scholars published the paper "Study on information system of health care 
services management in hospital". It was mainly about users' satisfaction of hospital services, which provided evaluation method and designed a framework for the management system of hospital services. The management system HSMS was taken as an example, and it was developed in order to improve the attract ability for hospitals.

Since 2006, several new ideas appeared in the research of this field. Two papers were published in 2006 , one of which was mainly about the feasibility and effectiveness of providing sex education online. Empirical study was conducted in Shanghai, and it was found that online education could be effective and useful in changing students' attitude on sexrelated issues. Accordingly, Internet, adolescents and attitude appeared to be the popular entities. The other paper mainly focused on the medical assistant system design.

The year of 2007 could be a milestone for the research of health information behavior in China. although there were still few papers published in total, which was five to be precise, the research topics seemed to be more substantial and profound. The first study online health community was conducted, which was call the peer support system. It intended to provide mental health information and other related consultancy for medical students. It was launched and run by students. It was an innovative strategy for health information service in China. This study was also of great value the development progress in this field. Another study on users' health information searching behavior was implemented. It was mainly about the group of elder people. A 3-hr training workshop was provide and participants had received guidance about where to get health information. Selected authorative websites were introduced to them. The result suggested that after the workshop, there were significant change of health information source for the participants. And health information seeking behavior could be greatly influenced by this kind of workshop.

There were similar research focuses in both 2007 and 2008, which included online health information seeking, online health education, and healthcare system and technology. Some new ideas and topics were brought forward in 2009. For example, the privacy protection appeared to be the popular topic for the first time. The amount of published papers in 2010 and 2011 were both below ten. There was an extremely close relationship between the main topics of the two years, which consisted of health information seeking, internet, internet search, access to healthcare, and breast cancer. Whereas, three new topic came into our vision since 2011, which were social media, self-management and trust. Moreover, the three topics remain the most popular topics in this field until now. Consequently, we would like to name the year of 2011 as the landmark of health information behavior. Not only because the persistent focuses appeared that year, but also the number of research papers began to increase rapidly.

(1)social media: as a new communication channel, social media has been used in health information seeking since 2011. Users-generated contents on health-related websites could enhance the sharing and communication of personal health status and knowledge. It is also helpful for health promotion. Additionally, most papers focus on pattern and influence of social media websites. Other related issues were also within our concerning, including privacy protection, information quality. Twitter was the most frequently used example when doing analysis on social medias.

(2)Self-management: it is one of the main purposes of health information seeking and other relative behaviors. Various technologies and devices of telemedicine were occupied for healthcare selfmanagement improvement. Evaluation method of their use and countermeasures were proposed.

(3)Trust: it is mainly about privacy protection and quality assurance of the health information, which weighs a lot for a sustainable development in the long term[3]. This topic also has several aspects to research, such as the assessment of information quality[4], users' satisfaction with online health information[5] and so on.

From the perspective of a whole spectrum, both topics about health information seeking and internet have been the main focuses for nearly ten years in this area. The two topics have gained much attention from researchers throughout the whole evolution progress, followed by other two topics, social media and trust. These two have a shorter period of popularity, which starts from 2011. However, they seem to be more popular right now than the two typical topics, health information seeking and internet. So the latter two emerged later, but have stronger potential for growth.

There is another notable phenomenon in the graph above. As a new concern in this area, machine learning came into scholars' view in 2017, and successfully became one of the most popular idea in one year. So it has appealed so much attention in a very short time, which is unique. With the use of machine learning in health information, feasible and practical information seeking and prediction have been achieved. Additionally, machine identification [6] and text mining[7] have also been realized. It is also useful for causal relationship analysis[8], 
tendency analysis[9] and information needs prediction[6][10]. In the contrary, some topic had an early emergence in this area. However, it is hard to become a hot topic. For example, the issue of accessibility is one of them. There is papers about access control [11] and access to health care [12] published all the time, but just few.

Consequently, according to the result of analysis by the Cortext, several conclusions could be made. First of all, the traditional scope of the research on health information behavior has been broadened during the past fifteen years. Both persistency and transiency exist within this period of time. Great changes happened to most of the hot topics. However, the topic of health information seeking has been the key point throughout the whole evolution progress without any change. This indicates that information seeking is the most important part and core content for the area of health information behavior. New method and technology for have been applied to health information seeking, for example social media and machine learning. Finally, the result suggests that cross-disciplinary research with new methods is the perpetual feature.

\subsection{Analysis on Clusters}

In order to understand the community structure and community networks of health information behavior, the word co-occurrence network has been gained with the use of VOS viewer, as shown in Figure 3. Keywords of the published papers have been represented as the nodes in this visualization. It can be found that there are 146 keywords in total which have appearances more than five times. Moreover, the size of the nodes is positively correlated to the times of appearance, while the thickness of lines has a positive correlation with the strength of words' co-occurrence.

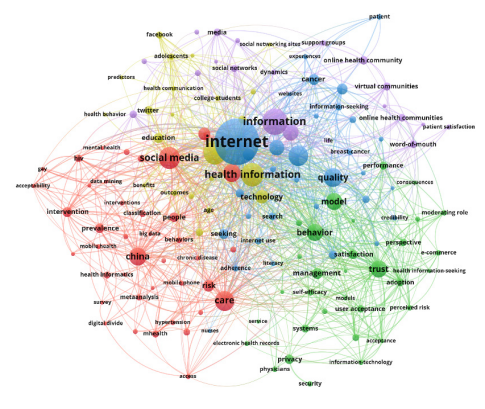

Figure.3. Word co-occurrence network of health information behavior

As a result, we have got to the conclusions below:
First of all, it is mainly about the online health information behavior in China, which is also our main focus of this study. This kind of topic consists of several issues, including health information mining on social media[13], identification and classification of the contents and opinions about health data on social media[6]. Moreover, it also contains research on users' opinions and behavior on social media[14], and the application of mobile health[15] and telemedicine[5]. Additionally, health informatics is also relatively involved [8];

Secondly, another important topic is mainly about trust of health information online. It refers to the accuracy, validity and related issues about the online health information. To be specific, sub-topics about this issue mainly include users' acceptance and adoption of online health information[16], ecommerce of online health information[17], quality evaluation of online health information[18], and the influential factors of the use of online health information;

Thirdly, research on health information seeking online is also within this scope. Health information acquisition[19], health information assessment[4], participants' roles and their decision-making are all related to the sub-topic[20][21];

Fourthly, support groups[22], social network structure and dynamics of online health communities have been approved to be relative topics[23]

To summarize, there are four aspects which have close relationship with health information behavior after the co-occurrence analysis on words, which are communication and relative behavior, trust, information seeking, structure and support.

\subsection{Analysis on Co-citation Network}

Figure 4 shows the co-cited network of these 412 papers. The size of the nodes represents its cited time, and the thickness of lines represents the strength of co-citation relationship.

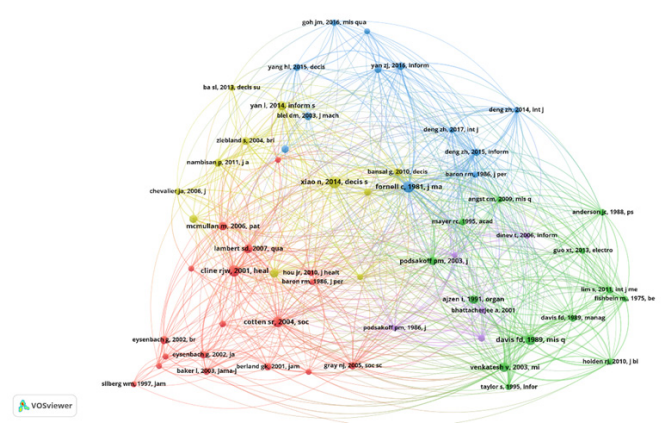


Figure.4. Co-citation Network of health information behavior

59 highly cited papers have been found to be the key articles of health information behavior, which have been cited no less than 7 times separately. This graph could suggest the importance of previous researches, which provide solid foundation for the followers. Here we have got five directions:

(1) Research on the features and conceptions of online health information seeking is the main focus which attracts numerous scholars to take part in it. They are mainly empirical study, which are mostly conducted based on relative conceptual models in other countries, such as the USA[24, 25,26];

(2) The model of users' acceptability is frequently applied for the development of online health information acceptance and adoption. For this direction, conclusions and extension have been made on the basis of traditional models, with the use of computer technology. In this way, the accuracy could be improved obviously[27, 28, 29];

(3) Structural equation model has been combined with TAM model, which could get improvement in the stability and applicable scope[30]. This kind of application is more suitable for the research on perceived value, perceived social support and other related factors, and questionnaires and surveys are usually used[31,32,33];

(4) Social support to online health community is the fourth part with strong concerning. Supports from the society is essential to the development of online health community. Many experiments have proved that emotional supports to patients will be of great importance for their recovery[34,35].

\section{Conclusions and Study Limitations}

The purpose of this review is to reveal the evolving progress of health information behavior. And we put our focus on the articles which have the participation of Chinese scholars in order to discuss about the specific situation of China. Since the data used in this paper was retrieved from the core database of WOS, papers which were not indexed in the database were excluded. Further research and study was expected to be continued by broadening the scope of data source.

\section{References}

[1] Lancet $T$. How to attain the ambitious goals for health reform in China[J]. Lancet, 2015, 386(10002):1419.
[2] Molassiotis A, Xu M. Quality and safety issues of webbased information about herbal medicines in the treatment of cancer[J]. Complementary Therapies in Medicine, 2004, 12(4):217-227.

[3] Liu Y, Liu F, Qu Q, et al. Evaluating the Satisfaction of Patients Undergoing Hair Transplantation Surgery Using the FACE-Q Scales[J]. Aesthetic plastic surgery, 2019: 1-7. [4] Guo W, Wang W, Xu D, et al. Evaluating the Quality, Content, and Readability of Online Resources for Failed Back Spinal Surgery[J]. Spine, 2019, 44(7): 494-502.

[5] Zhang H, Zhang R, Lu X, et al. Impact of Personal Trust Tendency on Patient Compliance Based on Internet Health Information Seeking[J]. Telemedicine and e-Health, 2019.

[6] Liu X, Liu X, Sun J, et al. Proactive Suicide Prevention Online (PSPO): Machine Identification and Crisis Management for Chinese Social Media Users With Suicidal Thoughts and Behaviors[J]. Journal of Medical Internet Research, 2019, 21(5): e11705.

[7] Wang X, Zhao K, Cha S, et al. Mining user-generated content in an online smoking cessation community to identify smoking status: A machine learning approach[J]. Decision Support Systems, 2019, 116: 26-34.

[8] Lin S, Zhang Q, Chen F, et al. Smooth Bayesian network model for the prediction of future high-cost patients with $\operatorname{COPD}[\mathrm{J}]$. International journal of medical informatics, 2019, 126: 147-155.

[9] Nan Y, Gao Y. A machine learning method to monitor China's AIDS epidemics with data from Baidu trends[J]. PloS one, 2018, 13(7): e0199697.

[10] Du L, Xia C, Deng Z, et al. A machine learning based approach to identify protected health information in Chinese clinical text[J]. International journal of medical informatics, 2018, 116: 24-32.

[11] Jiang S, Duan M, Wang L. Toward Privacy-Preserving Symptoms Matching in SDN-Based Mobile Healthcare Social Networks[J]. IEEE Internet of Things Journal, 2018, 5(3): 1379-1388.

[12] Wong W C W, Ho P S Y, Liang J, et al. Road to better health and integration: a Delphi study on health service models for Hong Kong migrants[J]. International journal for equity in health, 2014, 13(1): 127.

[13] Chen P, Chai J, Zhang L, et al. Development and application of a Chinese webpage suicide information mining system (sims)[J]. Journal of medical systems, 2014, 38(11): 88 .

[14] Liu X, Sun M, Li J. Research on gender differences in online health communities[J]. International journal of medical informatics, 2018, 111: 172-181.

[15] Jiang J, Zhu Q, Zheng Y, et al. Perceptions and Acceptance of mHealth in Patients With Cardiovascular Diseases: A Cross-Sectional Study[J]. JMIR mHealth and uHealth, 2019, 7(2): e10117.

[16] Zhao Y, Li K, Zhang L. A meta-analysis of online health adoption and the moderating effect of economic development level[J]. International journal of medical informatics, 2019.

[17] Wu T, Deng Z, Zhang D, et al. Seeking and using intention of health information from doctors in social media: The effect of doctor-consumer interaction[J]. International journal of medical informatics, 2018, 115: 106-113. 
[18] Zhang R, Lu X, Wu W, et al. Mature or Emerging? The Impact of Treatment-Related Internet Health Information Seeking on Patients' Trust in Physicians[J]. International journal of environmental research and public health, 2018, 15(9): 1855.

[19] Zhang X, Wen D, Liang J, et al. How the public uses social media WeChat to obtain health information in china: a survey study[J]. BMC medical informatics and decision making, 2017, 17(2): 66.

[20] Zhang X, Guo X, Lai K, et al. How does online interactional unfairness matter for patient-doctor relationship quality in online health consultation? The contingencies of professional seniority and disease severity[J]. European Journal of Information Systems, 2019, 28(3): 336-354

[21] Chan W S Y, Leung A Y M. Use of social network sites for communication among health professionals: systematic review[J]. Journal of medical Internet research, 2018, 20(3): e117.

[22] Zhang X, Liu S, Chen X, et al. Health information privacy concerns, antecedents, and information disclosure intention in online health communities[J]. Information \& Management, 2018, 55(4): 482-493.

[23] Yan L, Yan X, Tan Y, et al. Shared Minds: How Patients Use Collaborative Information Sharing via Social Media Platforms[J]. Production and Operations Management, 2019, 28(1): 9-26.

[24] Cotten S R , Gupta S S . Characteristics of online and offline health information seekers and factors that discriminate between them[J]. Social Science \& Medicine, 2004, 59(9):1795-1806.

[25] Cline R J W , Haynes K M . Consumer health information seeking on the Internet: the state of the $\operatorname{art}[\mathrm{J}]$. Health Educ Res, 2001, 16(6):671-692.

[26] Lambert S D , Loiselle C G . Health Information Seeking Behavior[J]. Qualitative Health Research, 2007, 17(8):1006-1019.

[27] Davis F D . Perceived Usefulness, Perceived Ease of Use, and User Acceptance of Information Technology[J]. MIS Quarterly, 1989, 13(3):319-340.

[28] Ajzen I . The theory of planned behavior[J]. Organizational Behavior \& Human Decision Processes, 1991, 50(2):179-211.

[29] Venkatesh V . User acceptance of information technology : Toward a unified view[J]. MIS Quarterly, 2003, 27.

[30] Gefen D , Straub K D W . Trust and TAM in Online Shopping: An Integrated Model[J]. MIS Quarterly, 2003, 27(1):51-90.

[31] Fornell C , Larcker D F . Evaluating Structural Equation Models with Unobservable Variables and Measurement Error[J]. Journal of Marketing Research, 1981, 18(1):39-50.

[32] Deng Z , Liu S , Hinz O . The health information seeking and usage behavior intention of Chinese consumers through mobile phones[J]. Information Technology \& People, 2015, 28(2):405-423.

[33] Yan Z, Wang T, Chen Y, et al. Knowledge sharing in online health communities: A social exchange theory perspective[J]. Information \& Management, 2016:S0378720616300040
[34] Ziebland S, Chapple A, Dumelow C, et al. How the internet affects patients' experience of cancer: A qualitative study[J]. BMJ (online), 2004, 328(7439):564.

[35] Yan L , Tan Y. Feeling Blue? Go Online: An Empirical Study of Social Support Among Patients[J]. Information Systems Research, 2014, 25(4):690-709. 